\title{
Efficacia e sicurezza del trattamento con un impianto sottocutaneo di octreotide in pazienti con acromegalia
}

\author{
Marco Centanni · Miriam Cellini
}

Pubblicato online: 11 marzo 2014

C) Springer International Publishing AG 2014

Commento a:

Efficacy and safety of an octreotide implant in the treatment of patients with acromegaly.

C. Chieffo, D. Cook, Q. Xiang and L.A. Frohman.

J Clin Endocrinol Metab 2013 October 98(10):40474054

Lo scopo principale del trattamento nei pazienti acromegalici è quello di riportare i livelli di GH e IGF-1 entro i valori di normalità. Sebbene l'escissione chirurgica dell'adenoma ipofisario GH-secernente rappresenti spesso il primo intervento terapeutico, la terapia medica trova molteplici indicazioni (pazienti in attesa di radioterapia o nei quali la chirurgia è stata inefficace o è controindicata, ecc.). L'octreotide, l'analogo della somatostatina più studiato e maggiormente utilizzato, è disponibile in due formulazioni: l'octreotide acetato e l'octreotide LAR a rilascio prolungato. Tuttavia, il trattamento giornaliero con octreotide acetato o circamensile con octreotide LAR genera fluttuazioni nella concentrazione ematica dell'analogo, non sempre è ben tollerato e/o causa effetti indesiderati quali l'ipertensione arteriosa e le colecistopatie [1].

Lo studio di fase 3 condotto da Chieffo et al. ha valutato l'efficacia e la tollerabilità comparativa di un impianto sottocutaneo di microsfere, rivestite da una capsula di idrogel, contenenti octreotide a lento rilascio. Un totale di 163 pazienti acromegalici già trattati con successo, per almeno tre mesi, con iniezioni mensili di octreotide LAR, è stato suddiviso in maniera randomizzata in due gruppi. I pazienti

M. Centanni $(\varangle) \cdot$ M. Cellini

Dipartimento di Scienze e Biotecnologie Medico-Chirurgiche,

U.O.C. di Endocrinologia, "Sapienza" Università di Roma,

Latina, Italia

e-mail: marco.centanni@uniroma1.it sono stati sottoposti rispettivamente a trattamento con impianto sottocutaneo nell' arto superiore di octreotide $(84 \mathrm{mg})$ o al trattamento con la formulazione LAR ogni 4 settimane $(10-40 \mathrm{mg})$, con un rapporto di 3 pazienti con impianto $(n=122)$ per ogni paziente in trattamento tradizionale $(n=41)$. Per un periodo di 24 settimane è stato eseguito il dosaggio di GH e IGF-1 e un questionario per la valutazione della qualità di vita è stato sottoposto ai pazienti (a 0,3 e 6 mesi).

Alla fine dello studio, nell' $86 \%$ dei pazienti con impianto sottocutaneo e nell' $84 \%$ di quelli in terapia con octreotide LAR sono stati ottenuti livelli adeguati e sovrapponibili di GH e IGF-1. La nuova formulazione ha però mostrato un migliore profilo farmacocinetico, con rilascio di octreotide continuo e costante, caratterizzato da un picco tra la $2^{\mathrm{a}}$ e la $4^{\mathrm{a}}$ settimana e una graduale riduzione dei livelli (fino al $48,6 \%$ ) dall' $8^{\mathrm{a}}$ alla $24^{\mathrm{a}}$ settimana, comunque mai inferiori a quelli ottenuti col trattamento tradizionale. Riguardo ai già descritti effetti collaterali riportati nei pazienti trattati con octreotide LAR (ipertensione arteriosa e colecistiti), nei pazienti con impianto sottocutaneo sono apparsi con minore frequenza, mentre più frequenti risultavano la diarrea e la cefalea; soltanto il $13 \%$ dei pazienti ha riportato una scarsa tollerabilità locale al dispositivo sottocutaneo, riconducibile principalmente a dolore, prurito e reazioni cutanee nel sito dell'impianto. Dall'analisi del questionario, inoltre, 1 ' $82,5 \%$ dei pazienti ha preferito il trattamento con dispositivo sottocutaneo rispetto alle iniezioni mensili.

In conclusione, questo studio dimostra che gli impianti sottocutanei di octreotide hanno un'efficacia terapeutica paragonabile a quella dell'octreotide LAR, avvalendosi però di un rilascio continuo del farmaco. Pertanto, questo nuovo dispositivo sottocutaneo può essere considerato un'opzione terapeutica aggiuntiva, specie nei pazienti candidati al trattamento prolungato con octreotide. 


\section{Bibliografia}

1. De Los Monteros AL, Carrasco CA, Albarrán AA et al (2014) The role of primary pharmacological therapy in acromegaly. Pituitary 17(1):4-10 